\title{
O EFEITO FÉRULA TEM INFLUÊNCIA NA OCORRÊNCIA DE FALHA E SOBREVIDA EM DENTES COM RESTAURAÇÕES RETIDAS POR PINO DE FIBRA? REVISÃO DE LITERATURA
}

\section{DOES FERULA EFFECT HAS ANY INFLUENCE ON THE OCCURRENCE OF FAILURE AND CLINICAL SURVIVAL OF FIBER-REINFORCED COMPOSITE POST-AND-CORE RESTORATIONS? LITERATURE REVIEW}

Francisco Ivison Rodrigues Limeira Doutor em Clínica Odontológica, Professor do

Curso de Odontologia da Faculdade

Presidente Antônio Carlos, Brasil.

E-mail: ivisonodontoce@hotmail.com

Diandra Costa Arantes

Doutora em Clínica Odontológica, Professora da Faculdade de Odontologia da Universidade

Federal do Pará, Brasil.

E-mail: arantesdiandra@yahoo.com.br

Daniel José Braga Dutra

Mestre em Clínica Odontológica, Professor da

Faculdade São Leopoldo Mandic Belo

Horizonte, Brasil.

E-mail: danielbd3@hotmail.com

\section{Resumo}

Este estudo teve por objetivo realizar uma revisão da literatura acerca da influência do efeito férula na ocorrência de falha e sobrevida clínica de restaurações retidas por pino de fibra. Foi realizada uma busca nas bases de dados PubMed/MEDLINE, Scopus e Cochrane Library. Foi utilizada uma estratégia de busca centrada nos termos relacionados ao DM e a PA, utilizando os seguintes termos MESH: "Ferrule", "Ferrule effect", "Fiber posts" e "Post and Core Technique". Artigos publicados até julho de 
2019 foram revisados. Após a busca, foi possível observar apenas 4 estudos clínicos investigam a influência do efeito férula na ocorrência de falha e sobrevida clínica de restaurações retidas por pino de fibra. Foi possível observar que a preservação de pelo menos uma parede coronária colaborou na diminuição da ocorrência de falhas e no aumento da sobrevida dos dentes restaurados com uso de pinos de fibra. Além do mais, a colocação de pinos de fibra também apresentou uma contribuição significativa nas taxas de falha e sobrevida. A taxa de sobrevida variou entre $72,5 \%$ e $95 \%$ nos grupos de dentes com férula e de $62,5 \%$ a $95,2 \%$ nos grupos sem férula. Contudo, apenas um estudo mostrou diferença na sobrevida dos dentes restaurados, com o efeito férula favorecendo a sobrevida. Assim, foi possível concluir que o efeito férula parece não influenciar significativamente na redução das taxas de falhas e no aumento da sobrevida de restaurações de dentes não vitais retidas por pino de fibra. No entanto, os estudos relacionados a essa temática ainda são poucos, fazendo-se necessários ensaios clínicos bem delineados.

Palavras-chave: Restauração Dentária Permanente; Técnica para Retentor Intrarradicular; Falha de Restauração Dentária; Taxa de Sobrevida.

\section{Abstract}

This study aimed to perform a literature review about the influence of the ferula effect on the occurrence of failure and clinical survival of fiber-reinforced composite post-andcore restorations. A search was performed in the PubMed / MEDLINE, Scopus and Cochrane Library databases. A search strategy centered on terms related to DM and PA was used, using the following MESH terms: "Ferrule", "Ferrule effect", "Fiber posts" and "Post and Core Technique". Articles published until July 2019 were reviewed. After the search, it was possible to observe only 4 clinical studies investigating the influence of the ferula effect on the occurrence of failure and clinical survival of fiber-reinforced composite post-and-core restorations. It was observed that the preservation of at least one coronary wall helped to reduce the occurrence of failures and increase the survival of teeth restored using fiber posts. In addition, fiber post placement also made a significant contribution to failure and survival rates. Survival rates ranged from $72.5 \%$ to $95 \%$ in the teeth with ferula groups and from $62.5 \%$ to $95.2 \%$ in the groups without ferula effect. However, only one study showed difference in survival of restored teeth, with the ferula effect favoring survival. Thus, it was possible to conclude that the ferula effect does not seem to significantly influence the reduction of failure rates and the increased survival of fiber-reinforced composite post-and-core restorations. However, there are still few studies related to this subject, requiring well-designed clinical trials.

Keywords: Dental Restoration Permanent; Post and Core Technique; Dental Restoration Failure; Survival Rate.

\section{Introdução}

Pinos de fibra são frequentemente usados para auxiliar na retenção da restauração de dentes não vitais comprometidos estruturalmente (ZICARI et al., 2013; NAUMANN et al., 2016). Em comparação com pinos de materiais mais rígidos, os 
pinos de fibra têm vantagens como seu módulo de elasticidade, que é semelhante ao da dentina, e capacidade de distribuir uniformemente o estresse ao longo do pino, cimento e dentina (ASSIF, 1994; SPAZZIN et al., 2009).

Diferentes fatores clínicos estão claramente relacionados ao sucesso e à sobrevida das restaurações de dentes não vitais retidas por pino de fibra, como o tipo e a posição do dente, sua relação com o antagonista, a presença de contatos interproximais, o tipo de restauração definitiva e, principalmente, a quantidade de estrutura coronal residual (MANCEBO et al., 2010; FERRARI et al., 2012; NAUMANN et al., 2012).

A presença de pelo menos $2 \mathrm{~mm}$ de dentina na porção coronal tem sido relatada como um dos fatores mais importantes para aumentar a resistência à fratura da restauração final e é conhecida como "efeito férula" (MANCEBO et al., 2010; FERRARI et al., 2012). Um colar de dentina circunferencial melhora o desempenho biomecânico do dente e está diretamente associado a uma maior resistência à fratura e, como consequência, uma maior sobrevivência, mas este último ainda precisa ser comprovado (MANCEBO et al. 2010; SAMRAN et al. 2013; WATANABE et al. 2012FIGUEIREDO et al., 2015). No entanto, falta um consenso sobre essas vantagens, pois os ensaios mecânicos e clínicos têm mostrados resultados conflitantes (VAGIDIACO et al., 2008; MANCEBO et al., 2010; FERRARI et al., 2012; FRAGOU et al., 2012; VALDIVIA et al., 2018).

Há evidências de alto nível que mostram que a ausência de parede coronal pode aumentar o risco de falha da restauração retida por pino, enquanto a influência do efeito férula ainda não está totalmente compreendida (YANG et al., 2015). Considerando a falta de consenso entre os estudos disponíveis, o estabelecimento de um protocolo clínico para dentes tratados endodonticamente com significativo comprometimento estrutural ainda é um desafio, sobretudo pelo fato de que outros fatores clínicos podem estar associados à sobrevida de dentes não vitais restaurados com o auxílio retentores intrarradiculares (SKUPIEN et al., 2016).

O objetivo deste estudo foi realizar uma revisão da literatura acerca da influência do efeito férula na ocorrência de falha e sobrevida clínica de restaurações retidas por pino de fibra. 


\section{Revisão de Literatura}

Foi realizada uma busca nas bases de dados PubMed/MEDLINE (https://www.ncbi.nlm.nih.gov/pubmed/), Scopus (https://www.scopus.com) e Cochrane Library (https://www.cochranelibrary.com). Foram utilizadas estratégias de buscas centradas nos termos relacionados à questão em estudo, utilizando a combinação dos seguintes termos MESH: "Ferrule", "Ferrule effect", "Fiber posts" e "Post and Core Technique". Com uso do operador booleano "AND".

Foram incluídos nesta revisão apenas ensaios clínicos que realizaram a comparação da sobrevida de restaurações retidas por pino de fibra em dentes tratados endodonticamente entre àqueles com e sem a presença de férula (colar de dentina coronal circunferencial de no mínimo $2 \mathrm{~mm}$ ). Não foram utilizados filtros que pudessem restringir o período de publicação do artigo. Todos os artigos recuperados nas buscas e publicados até julho de 2019 foram revisados. Foram excluídos estudos que não compararam a sobrevida da reabilitação de dentes tratados endodonticamente com e sem férula, revisões da literatura, estudos in vitro, análises de elementos finitos e estudos retrospectivos.

Cagidiaco et al. (2008) avaliaram se a quantidade de dentina coronal residual e a colocação de um pino de fibra de vidro pré-fabricado ou personalizado (anatômico) influenciavam na sobrevida, em três anos, de pré-molares tratados endodonticamente. Uma amostra de 60 pré-molares (345 pacientes) que necessitava de tratamento do canal radicular foi dividida de acordo com a quantidade de dentina deixada no nível coronal após o tratamento endodôntico e antes do preparo para o pino: G1 - quatro paredes coronais; G2 - três paredes coronais; G3 - duas paredes coronais; G4 - uma parede coronal; G5 - presença de férula (colar de dentina com 2 mm de altura); e G6 ausência de férula. Dentro de cada grupo, os dentes foram subdivididos aleatoriamente em três subgrupos $(n=20)$, definidos com base no procedimento restaurador: sem pino de fibra, pino de fibra pré-fabricado ou pino de fibra personalizado. Todos os dentes foram restaurados com uma coroa metalocerâmica. $A$ análise de regressão de Cox mostrou que a presença de retenção intrarradicular foi um fator significativo para a sobrevida das restaurações $(p<0,05)$. A redução do risco de falha foi maior nos dentes restaurados com pino de fibra pré-fabricado (taxa de risco $=0,1$ ) do que quando se usava pino de fibra personalizado (taxa de risco $=0,5$ ). 
Dentes com uma (taxa de risco $=0,3$ ), duas (taxa de risco $=0,2$ ) ou três (taxa de risco $=0,1)$ paredes coronais tiveram um risco de falha significativamente menor do que os dentes com o efeito férula. Riscos de falha semelhantes foram observados para os dentes sem nenhuma parede, independentemente da presença ou ausência do efeito férula $(p>0,05)$. As taxas de sobrevida para os grupos de dentes com férula e sem férula foram de $72,5 \%$ e $62,5 \%$, respectivamente ( $p>0,05)$. Assim, a colocação de pinos de fibra mostrou uma contribuição significativa para a sobrevida de pré-molares restaurados. Independentemente do procedimento restaurador, a preservação de pelo menos uma parede coronal reduziu significativamente o risco de falha, sem influência do efeito férula.

Bitter et al. (2009) avaliaram se a colocação de pino de fibra de vidro e a quantidade de dentina coronal residual afetam o tempo até a falha de restaurações de dentes tratados endodonticamente. Noventa pacientes com 120 dentes (anteriores, pré-molares e molares) foram selecionados. Três grupos $(n=40)$ foram definidos com base na quantidade de dentina coronal residual: G1- duas ou mais paredes coronais excedendo $2 \mathrm{~mm}$ acima do nível gengival; G2 - uma parede coronal excedendo $2 \mathrm{~mm}$ acima do nível gengival; e G3 - nenhuma parede excedendo $2 \mathrm{~mm}$ acima do nível gengival. Dentro de cada grupo, os dentes foram subdivididos em dois subgrupos de intervenção $(n=20)$ : restaurados com pino de fibra e restaurados sem pino de fibra. $A$ restauração final foi escolhida conforme cada situação clínica e perda de estrutura coronal. Após um período médio de observação de 32,4 meses, nos subgrupos sem pinos, as taxas de falha foram de $10 \%$, enquanto nos subgrupos com pinos, foram observadas taxas de falha de $7 \%(p=0,318)$. Nos grupos sem nenhuma parede, a colocação do pino de fibra afetou significativamente o tempo até a falha do total de restaurações $(p=0,029)$. Dentes sem retenção intrarradicular revelaram uma taxa de falha significativamente maior (31\%) em comparação com os dentes restaurados com pino de fibra (7\%). As taxas de sobrevida para os grupos de dentes com férula e sem férula foram de $95 \%$ e 95,2\%, respectivamente ( $p>0,05)$. Assim, concluíram que a colocação de pinos de fibra é eficaz para reduzir as falhas de restaurações de dentes tratados endodonticamente que não possuem remanescente coronal (acima de $2 \mathrm{~mm}$ ).

Mancebo et al. (2010) avaliaram se o tipo de dente e o efeito férula afetam significativamente a sobrevida de dentes tratados endodonticamente e restaurados 
com pinos de fibra de vidro. Uma amostra de 87 dentes (87 pacientes) foi restaurada usando pino de fibra de vidro (incisivos, caninos, pré-molares e molares). Foram definidos dois grupos experimentais, de acordo com a presença ou ausência de férula: dentes com férula (remanescente coronal $>2 \mathrm{~mm}$ de altura); e dentes sem férula (remanescente coronal $<2 \mathrm{~mm}$ de altura). Cada dente foi restaurado com uma coroa de metalocerâmica ou cerâmica pura e os pacientes foram reavaliados a cada seis meses. A taxa de sobrevida global, em três anos de acompanhamento, foi de 83,9\%. No grupo de dentes com férula, foi observada um taxa de falha de 6,67\% e de $26,20 \%$ no grupo sem férula $(p<0,05)$. De acordo com o tipo de dente, os incisivos foram os dentes com maior taxa de falhas $(73,52 \%)$, mas não houve diferença entre os quatro tipos de dentes. As taxas de sobrevida para os grupos de dentes com férula e sem férula foram de 93\%,3 e 73,8\%, respectivamente $(p<0,05)$. Os autores concluíram que em dentes tratados endodonticamente e restaurados com técnicas adesivas e pinos de fibra, a presença da férula aumenta a sobrevida clínica do dente.

Ferrari et al. (2012) analisaram a contribuição da dentina coronal remanescente e da colocação de pino de fibra de vidro pré-fabricado ou personalizado (anatômico) para a sobrevida em seis anos de pré-molares tratados endodonticamente. Um total de 345 pacientes participou do estudo, compondo uma amostra de 360 pré-molares, divididos em seis grupos $(n=60)$, definidos com base na quantidade de dentina deixada no nível coronal após o tratamento do canal radicular e antes do preparo para o pino: G1 - quatro paredes coronais; G2 - três paredes coronais; G3 - duas paredes coronais; G4 - uma parede coronal; G5 - presença de férula (colar de dentina circunferencial de pelo menos $2 \mathrm{~mm}$ de altura); e G6 - ausência de férula. Em cada grupo, os dentes foram subdivididos aleatoriamente em três subgrupos $(n=20)$, definidos com base no procedimento restaurador: pino de fibra pré-fabricado, pino de fibra personalizado ou sem pino de fibra. A restauração final foi realizada com coroa metalocerâmica e os pacientes foram convocados após um, seis, 12, 24, 36 e 72 meses para exame clínico e radiográfico. A análise de regressão de Cox revelou que a retenção da restauração com pino de fibra melhorou significativamente a sobrevida dos dentes $(p<0,001)$. O risco de falha foi menor nos dentes restaurados com pinos pré-fabricados (taxa de risco $=0,3$ ) do que com pinos personalizados (taxa de risco = 0,1 ). Dentes com uma (taxa de risco $=0,3$ ), duas (taxa de risco $=0,2$ ) e três (taxa de risco $=0.1)$ paredes coronais tiveram riscos de falha significativamente menores do 
que aqueles sem férula. Riscos de falha semelhantes existiram para dentes sem paredes coronais, independentemente da presença ou ausência de férula $(p=0,151)$. As taxas de sobrevida para os grupos de dentes com férula e sem férula foram de 92,6\% e 80,7\%, respectivamente (p>0,05). Assim, independentemente do procedimento restaurador, a preservação de pelo menos uma parede coronal reduz significativamente o risco de falha.

\section{Discussão}

Embora poucos estudos tenham sido localizados e incluídos nesta revisão da literatura, foi possível observar que a preservação de pelo menos uma parede coronária colaborou na diminuição da ocorrência de falhas e no aumento da sobrevida dos dentes restaurados com uso de pinos de fibra. Além disso, a colocação de pinos de fibra também apresentou uma contribuição significativa nas taxas de falha e sobrevida.

Os estudos de Cagidiaco et al. (2008) e Ferrari et al. (2012), mostraram que não existem diferenças quanto ao risco de falhas entre dentes com e sem a presença de férula. Cabe destacar, que os mesmos parecem resultar de um único ensaio clínico, publicados com diferentes tempos de acompanhamento. A taxa de sobrevida variou entre $72,5 \%$ e $95 \%$ nos grupos de dentes com férula e de $62,5 \%$ a $95,2 \%$ nos grupos sem férula. Contudo, apenas o estudo de Mancebo et al. (2010) mostrou diferença na sobrevida dos dentes restaurados, com o efeito férula favorecendo a sobrevida.

Quanto à possibilidade de fratura de restaurações retidas por pinos de fibra com ou sem dentina remanescente, a presença de férula tende a aumentar a resistência à ocorrência de fraturas (ICHIM et al., 2010). O efeito férula confere um efeito protetor, que resiste às forças funcionais, ao efeito de cunha dos pinos cônicos e às forças laterais geradas durante a inserção do pino (MANCEBO et al., 2010). A presença de férula pode estar relacionada à maior distribuição do estresse, pois análises de elementos finitos têm mostrado maiores valores de estresse, incluindo a região cervical, associada à ausência de ponteira (ERASLAN et al., 2009; PIERRISNARD et al., 2002). Nesse contexto, os pacientes devem ser informados da possibilidade de 
fraturas quando na presença de pouca ou nenhuma dentina coronária (ICHIM et al., 2010; MANCEBO et al., 2010).

Os estudos de Ferrari et al. (2012) e Cagidiaco et al. (2008) relataram inúmeros deslocamentos da coroa e todas as fraturas radiculares ocorreram nos dentes, com a estrutura coronal remanescente reduzida a uma ou duas paredes. Estudos biomecânicos relataram que a linha de cimentação e a interface adesiva são as regiões mais suscetíveis a falhas nas restaurações retidas por pinos de fibra (JULOSKI et al., 2013; ZHANG et al., 2015). Falhas como descementação e descolamento da coroa foram relatadas para ambos os grupos (com e sem férula) nos estudos incluídos. Portanto, é necessária uma técnica de cimentação correta, de acordo com as recomendações do fabricante, para obter uma interface adesiva adequada. A presença de férula, no entanto, pode impedir a exposição da linha de cimentação e, consequentemente, reduzir o risco de injúrias durante a mastigação e a possibilidade de microinfiltração (MANCEBO et al., 2010).

Juloski et al. (2012), analisando os resultados de pesquisas realizadas sobre as diferentes questões relacionadas ao efeito férula reportados por estudos in vitro, simulações em computador e ensaios clínicos, concluíram que a presença de uma férula de 1,5 a $2 \mathrm{~mm}$ tem um efeito positivo na resistência à fratura de dentes tratados endodonticamente. Se a situação clínica não permitir uma férula circunferencial, uma férula incompleta ainda é considerada a opção melhor do que a completa ausência de férula. Além disso, concluiu-se que quando da presença de férula durante a reabilitação, outros fatores como o tipo de pino, agentes de cimentação e restauração final, podem exercer menos impacto no desempenho da restauração retida por pino.

No entanto, é importante compreender que o efeito férula é apenas um dos requisitos importantes para que se tenha sucesso na restauração de dentes não vitais retidas por pino, que representa um sistema complexo afetado por vários outros fatores, como o tipo e a posição do dente, sua relação com o antagonista e a presença de contatos interproximais (AL-OMIRI et al., 2010; FERRARI et al., 2012; MANCEBO et al., 2010; NAUMANN et al., 2012).

\section{Considerações finais}


Diante do número limitado de estudos identificados e incluídos nesta revisão, foi possível concluir que o efeito férula parece não influenciar significativamente na redução das taxas de falhas e no aumento da sobrevida de restaurações de dentes não vitais retidas por pino de fibra, fazendo-se necessários ensaios clínicos com melhores delineamentos para estabelecimento de um protocolo clínico confiável. Contudo, a preservação de pelo menos uma parede coronária colabora na diminuição da ocorrência de falhas e no aumento da sobrevida dos dentes restaurados com uso de pinos de fibra, além do mais, a colocação de pinos de fibra também apresentou uma contribuição positiva nas taxas de falha e sobrevida.

\section{Referências}

AL-OMIRI, M. K.; MAHMOUD, A. A.; RAYYAN, M. R.; ABU-HAMMAD, O. Fracture resistance of teeth restored with post-retained restorations: an overview. J Endod, v. 36, n. 9, p. 1439-49, 2010.

ASSIF, D. Biomechanical considerations in restoring endodontically treated teeth. J Prosthet Dent, v. 71, n. 6, p. 565-67, 1994.

BITTER, K.; NOETZEL, J.; STAMM, O.; VAUDT, J.; MEYER-LUECKEL, H.; NEUMANN, K.; KIELBASSA, A. M. Randomized clinical trial comparing the effects of post placement on failure rate of postendodontic restorations: preliminary results of a mean period of 32 months. J Endod, v. 35, n. 11, p. 1477-82, 2009.

CAGIDIACO, M. C.; GARCÍA-GODOY, F.; VICHI, A.; GRANDINI, S.; GORACCI, C.; FERRARI, M. Placement of fiber prefabricated or custom made posts affects the 3-year survival of endodontically treated premolars. Am J Dent, v. 21, n. 3, p. 179-84, 2008.

ERASLAN, O.; AYKENT, F.; YUCEL, M. T.; AKMAN, S. The finite element analysis of the effect of ferrule height on stress distribution at post-and-core-restored all-ceramic anterior crowns. Clin Oral Investig, v. 13, n. 2, p. 223-7, 2009. 
FERRARI, M.; VICHI, A.; FADDA, G. M.; CAGIDIACO, M. C.; TAY, F. R.; BRESCHI, L.; POLIMENI, A.; GORACCI, C. A randomized controlled trial of endodontically treated and restored premolars. J Dent Res, v. 91, suppl. 7, p. 72s-8s, 2012.

FIGUEIREDO, F. E.; MARTINS-FILHO, P. R.; FARIA-E-SILVA, A. L. Do metal postretained restorations result in more root fractures than fiber post-retained restorations? A systematic review and meta-analysis. J Endod, v. 41, n. 3, p. 309-16, 2015.

FRAGOU, T.; TORTOPIDIS, D.; KONTONASAKI, E.; EVANGELINAKI, E.; IOANNIDIS, K.; PETRIDIS, H.; KOIDIS, P. The effect of ferrule on the fracture mode of endodontically treated canines restored with fibre posts and metal-ceramic or allceramic crowns. J Dent, v. 40, n. 4, p. 276-85, 2012.

ICHIM, I.; KUZMANOVIC, D. V.; LOVE, R. M. A finite element analysis of ferrule design on restoration resistance and distribution of stress within a root. Int Endod J, v. 39, n. 6, p. 443-52, 2006.

JULOSKI, J.; APICELLA, D.; FERRARI, M. The effect of ferrule height on stress distribution within a tooth restored with fibre posts and ceramic crown: a finite element analysis. Dent Mater, v. 30, n. 12, p. 1304-15, 2013.

JULOSKI, J.; RADOVIC, I.; GORACCI, C.; VULICEVIC, Z. R.; FERRARI, M. Ferrule effect: a literature review. J Endod, v. 38, n. 1, p. 11-9, 2012.

MANCEBO, J. C.; JIMENEZ-CASTELLANOS, E.; CANADAS, D. Effect of tooth type and ferrule on the survival of pulpless teeth restored with fiber posts: a 3-year clinical study. Am J Dent, v. 23, n. 6, p. 351-6, 2010. 
NAUMANN, M.; KOELPIN, M.; BEUER, F.; MEYER-LUECKEL, H. 10-year survival evaluation for glass-fiber-supported postendodontic restoration: a prospective observational clinical study. J Endod, v. 38, n. 4, p. 432-5, 2012.

NAUMANN, M.; NEUHAUS, K. W.; KOLPIN, M.; SEEMANN, R. Why, when, and how general practitioners restore endodontically treated teeth: a representative survey in Germany. Clin Oral Investig, v. 20, n. 2, p. 253-9, 2016.

PIERRISNARD, L.; BOHIN, F.; RENAULT, P.; BARQUINS, M. Corono-radicular reconstruction of pulpless teeth: a mechanical study using finite element analysis. $\mathbf{J}$ Prosthet Dent, v. 88, n. 4, p. 442-8, 2002.

SAMRAN, A.; EL BAHRA, S.; KERN, M. The influence of substance loss and ferrule height on the fracture resistance of endodontically treated premolars: an in vitro study. Dent Mater, v. 29, n. 12, p. 1280-6, 2013.

SKUPIEN, J. A.; LUZ, M. S.; PEREIRA-CENCI, T. Ferrule Effect: A Meta-analysis. JDR Clin Trans Res, v. 1, n. 1, p. 31-9, 2016.

SPAZZIN, A. O.; GALAFASSI, D.; MEIRA-JUNIOR, A. D.; BRAZ, R.; GARBIN, C. A. Influence of post and resin cement on stress distribution of maxillary central incisors restored with direct resin composite. Oper Dent, v. 34, n. 2, p. 223-9, 2009.

SALOMAO, P. E. A. et al. As Tecnologias de Informação e Comunicação (TIC) no ensino superior. Revista Multidisciplinar do Nordeste Mineiro, v. 1, 2018.

VALDIVIA, A. D. C. M.; RODRIGUES, M. P.; BICALHO, A. A.; VAN MEERBEEK, B.; SLOTEN, J. V.; PESSOA, R. S. E.; SOARES, C. J. Biomechanical effect of ferrule on incisors restored with a fiberglass post and lithium-disilicate ceramic crown after thermal cycling and fatigue loading. J Adhes Dent, v. 20, n. 2, p. 133-42, 2018. 
WATANABE, M. U.; ANCHIETA, R. B.; ROCHA, E. P.; KINA, S.; ALMEIDA, E. O.; FREITAS, A. C. J. R.; BASTING, R. T. Influence of crown ferrule heights and dowel material selection on the mechanical behavior of root-filled teeth: a finite element analysis. J Prosthodont, v. 21, n. 4, p. 304-11, 2012.

YANG, A.; LAMICHHANE, A.; XU, C. Remaining coronal dentin and risk of fiberreinforced composite post-core restoration failure: a meta-analysis. Int J Prosthodont, v. 28, n. 3, p. 258-64, 2015.

ZHANG, Y. Y.; PENG, M. D.; WANG, Y. N.; LI, Q. The effects of ferrule configuration on the anti-fracture ability of fiber post-restored teeth. J Dent, v. 43, n. 1, p. 117-25, 2015.

ZICARI, F.; VAN MEERBEEK, B.; SCOTTI, R.; NAERT, I. Effect of ferrule and post placement on fracture resistance of endodontically treated teeth after fatigue loading. $\mathbf{J}$ Dent, v. 41, n. 3, p. 207-15, 2013. 